\title{
Emerging trend of candidiasis with special reference to biofilm production
}

\author{
N Rajkumar ${ }^{1}$, S Mohan², S Khaja Moinuddin ${ }^{3 *}$
}

\begin{abstract}
${ }^{1}$ Department of Microbiology, Dhanalakshmi Srinivasan Medical College, Perambalur-621113, Tamil Nadu, INDIA.

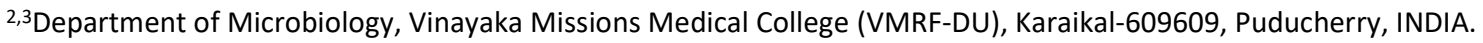
Email: moinnewlook@gmail.com
\end{abstract}

\section{$\underline{\text { Abstract }}$}

\begin{abstract}
Background: Non albican Candida (NAC) recently gained recognition as emerging pathogen because of its high virulence. Biofilm production among non albican candida poses clinical concerns as it enhances tolerance to antifungal drugs and it is not entirely understood the mechanism of biofilm tolerance to antifungals. Hence, the present study was aimed to determine the isolation rate of NAC from clinical specimens and biofilm production. Materials and methods: This was a descriptive, cross-sectional study, conducted over seven months in the department of microbiology at Dhanlakshmi Sreenivasan medical college and hospital. Candida isolated from clinical specimens were identified upto species level by HiMedia CHROM agar ${ }^{\circledR}$. Biofilm production was detected by tube method. Results: A total of 137 Candida species were isolated from various clinical specimens during the study period. Out of 137 Candida isolates, 77(56.20\%) were identified as Candida albicans, and the remaining 60(43.80\%) were non albican Candida (NAC). Among NAC, C. tropicalis (19.71\%) was the predominant isolate. In our study, 58(42.34\%) isolates were found to be biofilm producers. Overall biofilm formation was found to be high among NAC 37(63.80\%) compared to 21(36.20\%) C.albicans. Among NAC, C. tropicalis was observed as a predominant biofilm producer. Conclusion: Candida tropicalis was found to be common among non albican candida. Biofilm production was predominant among non albican candida compared to C.albicans.
\end{abstract}

Key Words: Biofilms, Non albican candida, Tube method.

*Address for Correspondence:

Dr Shaik Khaja Moinuddin, Department of Microbiology, Vinayaka missions medical college (VMRF-DU), Karaikal-609609, Puducherry., INDIA.

Email: moinnewlook@gmail.com

Received Date: 03/03/2020 Revised Date: 08/05/2020 Accepted Date: 15/06/2020

DOI: https://doi.org/10.26611/10081511

This work is licensed under a Creative Commons Attribution-NonCommercial 4.0 International License. $(\boldsymbol{\alpha c )})$ EY-NC

\begin{tabular}{|l|l|}
\hline \multicolumn{2}{|c|}{ Access this article online } \\
\hline Quick Response Code: & Website: \\
& www.medpulse.in \\
\cline { 2 - 2 } & \\
& Accessed Date: \\
& 02 July 2020 \\
\hline
\end{tabular}

\section{INTRODUCTION}

Over the past two decades, candida infections have been intensified by increasing the common usage of multiple medical implant systems especially in patients with immunological deficiencies. ${ }^{1}$ The growing prevalence of opportunistic candidiasis is based entirely on the frequent presence of Candida species in the human body surface's normal ecological niche. Therefore, this situation also encourages close interactions between Candida species and most embedded medical devices and the host surface leading to acute, persistent, or recurring infections. ${ }^{2}$ Until recently, C. albicans has been cited as the most common species which causes most candidiasis cases. However, multiple studies in just the last few decades have recorded a radical change from a preponderance of $\mathrm{C}$. albicans to non albicans Candida species (NAC), such as C. tropicalis, and C. glabrata, C. krusei. ${ }^{3}$ Disease causing nature of Candida species is connected to certain virulence factors as with the ability to escape host immune response, adherence, the formation of biofilms (on host tissue and medical devices) and the production of tissue-damaging hydrolytic enzymes such as proteases, phospholipases, and hemolysin. ${ }^{4}$ Current data indicate that the development of the disease by Candida species is connected to increasing biofilms production. Biofilm production helps the organism to tolerate or overcome the process of host 
defense and its disruptive effect and it helps the organism to survive and to act as a reservoir and persistent source of infection, as well as to establish antimicrobial resistance. Biofilm development poses clinical concerns as it enhances tolerance to antifungal drugs and it is not entirely understood the mechanism of biofilm tolerance to antimicrobials. ${ }^{5}$ Though considerable research has been done to identify these pathogenic features in C. albicans, comparably less of NAC species is known. Hence, the present study was aimed to determine the isolation rate of NAC from clinical specimens and biofilm production.

\section{MATERIALS AND METHODS}

This was a cross sectional study conducted over seven months in the department of microbiology at Dhanlakshmi Sreenivasan medical college and hospital. During the study period. The various clinical specimens were collected and processed as per the standard microbiological procedures. The Candida isolates which were obtained were further speciated by inoculating on chromogenic medium, HiMedia CHROM agar ${ }^{\circledR}$. Chromogenic substances in media help in the rapid identification of the Candida species, based on the reactions between the specific enzymes of the different species and the chromogenic substances. As per the color code which is given with the chromogenic media, speciation was performed.

Detection of biofilm formation by tube Method

A loopful of organisms from the surface of the SDA plate was inoculated into a tube containing $10 \mathrm{ml}$ of Sabouraud
Dextrose Broth supplemented with glucose. The tubes were incubated at $35^{\circ} \mathrm{C}$ for 48 hours. After incubation, the culture supernatants were decanted and the tubes were washed with phosphate buffer saline $(\mathrm{pH} 7.3)$ and the dried tubes were stained with $1 \%$ Safranin. Excess stain was removed by washing with de-ionized water. Tubes were then dried by positioning them invertedly. Tubes were then observed for biofilm formation. Biofilm formation was considered positive when a visible film lined the wall of the test tube.

Ethical committee approval was taken from Institution.

\section{RESULTS}

A total of 137 Candida species were isolated from various clinical specimens during the study period. Out of 137 Candida isolates, 77(56.20\%) were identified as Candida albicans, and the remaining 60(43.80\%) were non albican Candida (NAC). Among NAC, C. tropicalis (19.71\%) was the predominant isolate followed by $\mathrm{C}$. parapsilosis (13.14\%), C. krusie (8.76\%), and C. glabrata(2.19\%). Although Candida albicans were the found to be the common species isolated from various specimens, the number is not much high as compared to NAC. C. tropicalis was frequently isolated from urine samples followed by sputum, pus, and blood. A preponderance of C. parapsilosis was in pus samples followed by sputum, urine, and vaginal swabs. C. krusie was isolated from commonly from urine. Three strains of C.glabrata were isolated and all were from vaginal swabs ( Table.1)

\begin{tabular}{cccccc}
\multicolumn{7}{c}{ Table 1: Distribution of Candida species in clinical specimens } \\
\hline Specimens & C.albicans & C. tropicalis & C.parapsilosis & C.krusie & C.glabrata \\
\hline Sputum & 17 & 5 & 5 & 2 & - \\
Urine & 10 & 19 & 3 & 8 & - \\
Pus & 11 & 1 & 8 & - & - \\
Blood & 12 & 1 & - & 2 & - \\
Vaginal swabs & 10 & - & 2 & - & 3 \\
Ear & 8 & - & - & - & - \\
Eye & 7 & 1 & - & - & - \\
Body fluids & 2 & - & - & - & - \\
\hline Total & $77(56.20 \%)$ & $27(19.71 \%)$ & $18(13.14 \%)$ & $12(8.76 \%)$ & $3(2.19 \%)$ \\
\hline
\end{tabular}

In our study, $58(42.34 \%)$ isolates were found to be biofilm producers. Overall biofilm formation was found to be high among NAC $37(63.80 \%)$ compared to $21(36.20 \%)$ C.albicans. Among NAC, C. tropicalis was observed as a predominant biofilm producer.

Table 2: Biofilm formation among Candida species

\begin{tabular}{ccc}
\hline Candida species & Biofilm positive & Biofilm negative \\
\hline Candida albicans & 21 & 48 \\
C. tropicalis & 25 & 6 \\
C.parapsilosis & 7 & 13 \\
C.krusie & 3 & 10 \\
C.glabrata & 2 & 2 \\
\hline Total=137 & $\mathbf{5 8 ( 4 2 . 3 4 \% )}$ & $\mathbf{7 9 ( 5 7 . 6 6 \% )}$ \\
\hline
\end{tabular}




\section{DISCUSSION}

Candida species are found to be normal flora, and normal innate immunity interruptions are required to act as potential pathogens. Candida species have become emerging pathogens in patients such as immunocompromised, elderly, receiving antibacterial and intensive chemotherapy for cancer, or are undergoing invasive treatment. ${ }^{6}$ The present study aimed to speciate non albican candiada from various clinical specimens. Although Candida albicans remained as a predominant species, non albican candida are not very far to outnumber C.albicans. This is similar to the studies conducted previously. As per Kathy Montes et al., C. albicans was the frequently isolated species, with the remaining nonAlbicans representing $57 \%$ to the number. ${ }^{7} \mathrm{C}$. albicans preponderance compared with NAC species, vary between countries. However various studies showed the preponderance of the NAC..$^{8,9}$ The present study observed the changing pattern of Candida infections towards species NAC. A shift could be due to an advanced detection accuracy of Candida species from non-Albicans or a progressive change in prevalence. Our finding is consistent with an analysis carried out by Ghazi et al., which shows a changeover of Candida albicans towards non-Albicans Candida species. ${ }^{10}$ In our study, the second predominant Candida species was found to be C. tropicalis. This is in line with the results of the previous study. Das et al. ${ }^{9}$ The results of Uh et al. showed a preponderance of $C$. parapsilosis among NAC. ${ }^{11}$ Mohandas and Balla showed the second common Candida species from clinical species was C. krusei, followed by $\mathrm{C}$ tropicalis. ${ }^{12} \mathrm{Chi}$ et al., noticed C. glabrata as the second common pathogen followed by C. tropicalis. ${ }^{13}$ The effect of the different seasons, geographic, environmental differences of the fungi belonging to the Candida genus is believed to have resulted in these slight differences in the isolation rate. ${ }^{14}$ Biofilms are specific and organized communities of cells under the control of signaling molecules, rather than random accumulations of cells resulting from cell division. By evading host immune mechanisms, resisting antifungal treatment and withstanding competitive pressure from other organisms, biofilms can help maintain the role of fungi as pathogen In our study, biofilm producing Candida were analyzed by tube method. Analysis of our results showed that biofilm production was more common among NAC species. This is in agreement with the previous studies. ${ }^{15,16}$ Further we evaluated the associations between Candida species and the biofilm production of isolates. Candida obtained from the urine and blood samples were found to be typically biofilm producers. Urine isolates presenting intense biofilm production might be associated with the use of the urinary catheter, or be originated from systemic candidiasis and not from urinary tract infections since the isolates from invasive infections tend to produce more biofilm than those from non-invasive infections. ${ }^{17} \mathrm{In}$ our study, biofilm production was noticed in all Candida species isolated from clinical specimens. C.tropicalis was found to be the predominant biofilm producing organism. This finding is similar to Ariane Bruder-Nascimento et $a l .{ }^{18}$ As per their study, among biofilm producers,C. tropicalis showed the highest intensity of biofilm production. Further, a study by Shin et al., found C. tropicalis as the species with the highest percentages of biofilm positivity, while C. albicans, ranked third or fourth among the biofilm-positive isolates. ${ }^{19}$ However, our study results are not in line with the study conducted by Munmun B. Marak and Biranthabail Dhanashree. According to them, all strains of C.parapsilosis were biofilm producers and C. tropicalis was least. ${ }^{20}$ Nonalbicans cannot be ignored as just a contaminants or commensals of nonpathogenic nature. Research on predominant Candida species along with their virulence factors in a particular setup is really an important tool for demonstrating the relationship between Candida species and infection. The significant changes of Candida isolation from different clinical specimens have made it imperative to address Candida species that produce virulence factors for diagnosis. ${ }^{21}$ The present study has a few limitations. First, Candida species isolated from various samples were not subjected to antifungal susceptibility testing. Secondly, the Biofilm testing technique may have affected the findings, as we did not test biofilm in tandem with Candida biofilms' metabolic activity.

\section{CONCLUSION}

In our study, Candida tropicalis was found to be common among non albican candida. Biofilm production was predominant among non albican candida compared to C.albicans. By acting as a source for reinfection, biofilm development may play a crucial role in clinical outcomes. Timely screening of Candida biofilm formation may be essential to assess the magnitude of the infection and to make clinical decisions.

\section{REFERENCE}

1. Al-Fattani MA, Douglas LJ. Penetration of Candida biofilms by antifungal agents. Antimicrob Agents Chemother 2004;48:3291-97.

2. Kojic EM, Darniche RO. Candida infections of medical devices. Clin Microbiol Rev 2004;5:255-67.

3. Latha R, Sasikala R, Muruganandam RN, Babu RY. Study on the shifting patterns of Non Candida albicans Candida in lower respiratory tract infections and evaluation of the CHROMagar in identification of the Candida species. $\mathrm{J}$ Microbiol Biotech Res. 2011;1:113-9.

4. Silva S, Negri M, Henriques M, Oliveira R, Williams DW, Azeredo J. Adherence and biofilm formation of non- 
Candida albicans Candida species. Trends in microbiology. 2011 May 1;19(5):241-7.

5. Nett, J. E., Sanchez, H., Cain, M. T., Ross, K. M. and Andes, D. R. Interface ofCandida albicansbiofilm matrixassociated drugresistance and cell wall integrity regulation.Eukaryot Cell10. 2011. 1660-1669.

6. Mohandas V, Ballal M. Distribution of Candida species in different clinical samples and their virulence: biofilm formation, proteinase and phospholipase production: a study on hospitalized patients in southern India. Journal of global infectious diseases. $2011 \mathrm{Jan} ; 3(1): 4$.

7. Montes K, Ortiz B, Galindo C, Figueroa I, Braham S, Fontecha G. Identification of Candida Species from Clinical Samples in a Honduran Tertiary Hospital. Pathogens. 2019 Dec;8(4):237.

8. Kaur R, Dhakad MS, Goyal Kumar RR. Emergence of non-albicans Candida species and antifungal resistance in intensive care unit patients. Asian Pac J Trop Biomed. 2016;6:455-60.

9. Das KH, Getso MI, Azeez-Akande O. Distribution of Candida albicans and non-albicans Candida in clinical samples and their intrinsic biofilm production status. Int $\mathbf{J}$ Med Sci Public Health. 2016;5:2443-2244. doi: 10.5455/ijmsph.2016.26042016491.

10. Ghazi S, Rafei R, Osman M, Safadi EI, Mallat H, Papon $\mathrm{N}$, et al. The epidemiology of Candida species in the Middle East and North Africa. J Mycol Med. 2019;29:245-252. doi: 10.1016/j.mycmed.2019.07.006.

11. Uh Y, Jang IH, Yoon KJ, Kim HY. Isolation trend and antifungal susceptibility of Candida species isolated from blood cultures. Korean J Infect Dis. 2001;33:186-193.

12. Mohandas V, Ballal M. Distribution of Candida Species in different clinical samples and their virulence: Biofilm formation, proteinase and phospholipase production: A study on hospitalized patients in Southern India. J Glob Infec Dis. 2011;3:4-8. doi: 10.4103/0974-777X.77288.
13. Chi H-W, Yang Y-S, Shang S-T, Chen K-H, Yeh K-M, Chang F-Y, et al. Candida albicans versus non-albicans bloodstream infections: The comparison of risk factors and outcome. J Microbiol, Immun Infec. 2011;44:369e375.

14. Kim GY, Jeon JS, Kim JK. Isolation frequency characteristics of Candida species from clinical specimens. Mycobiology. 2016 Jun 1;44(2):99-104.

15. Girish-Kumar CP, Menon T. Biofilm production by clinical isolates of Candida species. Med Mycol 2006;44:99-101.

16. Muni S, Menon S, Chande C, Gohi A, Chowolhary A, Joshi A. Candida biofilm. Bombay Hosp J 2012;54(1):19 23

17. Kuhn DM, Chandra J, Mukherjee PK, Ghannoum MA. Comparison of biofilms formed by Candida albicans and Candida parapsilosis on bioprosthetic surfaces. Infection and immunity. 2002 Feb 1;70(2):878-88.

18. Bruder-Nascimento A, Camargo $\mathrm{CH}$, Mondelli AL, Sugizaki MF, Sadatsune T, Bagagli E. Candida species biofilm and Candida albicans ALS3 polymorphisms in clinical isolates. Brazilian journal of Microbiology. 2014 Dec;45(4):1371-7.

19. Shin JH, Kee SJ, Shin MG, Kim SH, Shin DH, Lee SK, Suh SP, Ryang DW. Biofilm production by isolates of Candida species recovered from nonneutropenic patients: comparison of bloodstream isolates with isolates from other sources. Journal of Clinical Microbiology. 2002 Apr $1 ; 40(4): 1244-8$.

20. Marak MB, Dhanashree B. Antifungal susceptibility and biofilm production of Candida spp. isolated from clinical samples. International journal of microbiology. 2018;2018.

21. Agwan V, Butola R, Madan M. Comparison of biofilm formation in clinical isolates of Candida species in a tertiary care center, North India. Indian J Pathol Microbiol 2015;58:475-8

\section{Source of Support: None Declared} Conflict of Interest: None Declared 